\title{
Refining the role of BRCA1 in combating oxidative stress
}

Jeffrey R Marks

\begin{abstract}
The BRCA1 hereditary susceptibility gene has been studied in great depth, befitting its clear role in promoting basal type breast cancer and serous type ovarian (fallopian tube) cancer in women carrying germline mutations. The BRCA1 protein has long been implicated in maintaining genome integrity through DNA repair processes. However, a number of studies have demonstrated that BRCA1 is also involved in the response to oxidative stress. A recent paper by Gorrini and colleagues extends our mechanistic understanding of how BRCA1 regulates this pathway. The relative contribution of this activity in BRCA1-associated tumorigenesis and DNA damage response remains unknown.
\end{abstract}

Germline BRCA1 mutation leads to the pronounced and specific phenotype of serous ovarian and basal breast cancer $[1,2]$. There is also strong evidence indicating that BRCA1 mutated cancers are particularly susceptible to genotoxic and oxidative agents such as PARP (poly ADP ribose polymerase) inhibitors and platinum [3]. Both of these properties of BRCA1 are thought to be associated with mechanisms of DNA repair, including homologous recombination, non-homologous end-joining, nucleotide excision repair, and transcription-coupled repair. However, an equally plausible explanation for BRCA1-associated biological effects may lie in its control over the oxidative stress response.

The response to oxidative stress is in large part regulated by a master transcription factor, NRF2 (NFE2L2), that induces expression of a large set of genes that act to restore redox balance and mitigate the damage from reactive oxygen species (ROS). NRF2 activity is controlled post-translationally by an E3 ubiquitin ligase, KEAP1. The KEAP1 protein senses oxidative stress by

Correspondence: marks003@mc.duke.edu

Department of Surgery, Division of Surgical Sciences, Duke University, Durham, NC 27710, USA undergoing a conformational change that prevents association with NRF2, allowing NRF2 to accumulate and translocate to the nucleus, thereby inducing transcription via anti-oxidant response elements located in a large set of genes that participate in balancing the redox state and repairing oxidative damage. This pathway appears central in maintaining oxidative homeostasis in many types of cells [4]. Further, somatic mutations in NRF2 pathway genes play a role in certain human cancers [5].

BRCA1 had been implicated previously in this cascade, initially by Bae and colleagues [6], who found that wildtype BRCA1 induced the transcription of a number of genes in the anti-oxidant response pathway conferring relative resistance to oxidative stress. Evidence was presented implicating increased activity of NRF2; however, NRF2 was not an obvious transcriptional target of BRCA1. The recent paper by Gorrini and colleagues fills in the missing mechanistic detail, demonstrating that BRCA1 can physically associate with NRF2 and this prevents binding and ubiquitination by KEAP1 [7], thus stabilizing NRF2 and activating downstream target genes and the anti-stress response. BRCA1 itself may be a transcriptional target of NRF2 and this could serve as a positive feedback loop to enhance the response to oxidative stress. Importantly, in BRCA1 deficient mammary cells, activation of NRF2 through small interfering RNA inhibition of KEAP1 rescued the survival defect associated with loss of BRCA1 and restored ROS levels, indicating that this mechanism is physiologically relevant. Overall, these data are consistent with previous reports $[8,9]$ and extend our biochemical understanding of BRCA1 in these processes.

Of note is another hereditary susceptibility gene that also appears to regulate the same pathway. PALB2 (partner and localizer of BRCA2; also termed Fanconi Anemia complementation group $\mathrm{N}$ ) is a major BRCA2 binding partner (and a relatively minor BRCA1 interactor) that is also mutated in familial breast and pancreatic cancer [10]. Ma and colleagues [11] recently showed that wild-type PALB2 binds to the E3 ligase KEAP1, thus preventing 
NRF2 from undergoing ubiquitination and degradation, resulting in the same net effect as wild-type BRCA1 in regulating oxidative homeostasis. It is unknown whether or how BRCA2 participates in this complex or how PALB2 activity may be regulated in response to stress. The relative contribution of BRCA1 versus PALB2/ BRCA2 in controlling this pathway is also unknown.

Like many other central response pathways, oxidative stress is modulated by a myriad of positive and negative signals that are transduced primarily via the levels of NRF2 in the nucleus. That BRCA1 and BRCA2 (by inference from PALB2) may both be involved in regulating this signal strongly implicates this pathway in the function of these hereditary cancer genes. By function, I specifically refer to both the cancer-promoting properties and the increased susceptibility of these cancers to genotoxic and oxidative agents, an intense focus of therapeutic testing and development [12].

Considering BRCA1 by itself, many hundreds of proteins have been shown to physically or genetically interact with it, implicating it in many pathways that could be considered fundamental to cancer development and drug response [13]. Which of these pathways is most important for the molecular pathophysiology of BRCA1 or is it a combination of several (for example, homologous recombination plus oxidative stress) that leads to the high penetrance of cancer and the sensitivity to DNA damaging agents associated with its loss of function? Further, we are still no closer to understanding the tissue-specific nature of the hereditary syndrome characterized by the selective neoplastic transformation of luminal progenitor cells in the breast and epithelial cells in the fimbria of the fallopian tube. One or more of these BRCA1-associated pathways will eventually forge an important mechanistic link between these two susceptible cell types.

Abbreviation

ROS: Reactive oxygen species.

\section{Competing interests}

The author declares that he has no competing interests.

Acknowledgement

The author thanks Jen-Tsan Chi for discussion and critical reading of the manuscript.

Published: 05 Dec 2013

\section{References}

1. Lakhani SR, Manek S, Penault-Llorca F, Flanagan A, Arnout L, Merrett S, McGuffog L, Steele D, Devilee P, Klijn JG, Meijers-Heijboer H, Radice P, Pilotti S, Nevanlinna H, Butzow R, Sobol H, Jacquemier J, Lyonet DS, Neuhausen SL, Weber B, Wagner T, Winqvist R, Bignon YJ, Monti F, Schmitt F, Lenoir G, Seitz S, Hamman U, Pharoah P, Lane G, et al: Pathology of ovarian cancers in BRCA1 and BRCA2 carriers. Clin Cancer Res 2004, 10:2473-2481.

2. Lee E, McKean-Cowdin R, Ma H, Spicer DV, Van Den Berg D, Bernstein L, Ursin G: Characteristics of triple-negative breast cancer in patients with a BRCA1 mutation: results from a population-based study of young women. J Clin Oncol 2011, 29:4373-4380.
3. Johnson N, Johnson SF, Yao W, Li YC, Choi YE, Bernhardy AJ, Wang Y, Capelletti M, Sarosiek KA, Moreau LA, Chowdhury D, Wickramanayake A, Harrell MI, Liu JF, D'Andrea AD, Miron A, Swisher EM, Shapiro Gl: Stabilization of mutant BRCA1 protein confers PARP inhibitor and platinum resistance. Proc Natl Acad Sci U S A 2013, 110:17041-17046.

4. Nguyen T, Nioi P, Pickett CB: The Nrf2-antioxidant response element signaling pathway and its activation by oxidative stress. J Biol Chem 2009, 284:13291-13295

5. Shibata T, Ohta T, Tong Kl, Kokubu A, Odogawa R, Tsuta K, Asamura H, Yamamoto M, Hirohashi S: Cancer related mutations in NRF2 impair its recognition by Keap1-Cul3 E3 ligase and promote malignancy. Proc Natl Acad Sci U S A 2008, 105:13568-13573.

6. Bae I, Fan S, Meng Q, Rih JK, Kim HJ, Kang HJ, Xu J, Goldberg ID, Jaiswal AK, Rosen EM: BRCA1 induces antioxidant gene expression and resistance to oxidative stress. Cancer Res 2004, 64:7893-7909.

7. Gorrini C, Baniasadi PS, Harris IS, Silvester J, Inoue S, Snow B, Joshi PA, Wakeham A, Molyneux SD, Martin B, Bouwman P, Cescon DW, Elia AJ, Winterton-Perks Z, Cruickshank J, Brenner D, Tseng A, Musgrave M, Berman HK, Khokha R, Jonkers J, Mak TW, Gauthier ML: BRCA1 interacts with Nrf2 to regulate antioxidant signaling and cell survival. J Exp Med 2013, 210:1529-1544.

8. Saha T, Rih JK, Rosen EM: BRCA1 down-regulates cellular levels of reactive oxygen species. FEBS Lett 2009, 583:1535-1543.

9. Martinez-Outschoorn UE, Balliet R, Lin Z, Whitaker-Menezes D, Birbe RC, Bombonati A, Pavlides S, Lamb R, Sneddon S, Howell A, Sotgia F, Lisanti MP: BRCA1 mutations drive oxidative stress and glycolysis in the tumor microenvironment: implications for breast cancer prevention with antioxidant therapies. Cell Cycle 2012, 11:4402-4413.

10. Tischkowitz M, Xia B: PALB2/FANCN: recombining cancer and Fanconi anemia. Cancer Res 2010, 70:7353-7359.

11. Ma J, Cai H, Wu T, Sobhian B, Huo Y, Alcivar A, Mehta M, Cheung KL, Ganesan S, Kong AN, Zhang DD, Xia B: PALB2 interacts with KEAP1 to promote NRF2 nuclear accumulation and function. Mol Cell Biol 2012, 32:1506-1517.

12. De Lorenzo SB, Patel AG, Hurley RM, Kaufmann SH: The elephant and the blind men: making sense of PARP inhibitors in homologous recombination deficient tumor cells. Front Oncol 2013, 3:228.

13. Woods NT, Mesquita RD, Sweet M, Carvalho MA, Li X, Liu Y, Nguyen H, Thomas CE, Iversen ES Jr, Marsillac S, Karchin R, Koomen J, Monteiro AN: Charting the landscape of tandem BRCT domain-mediated protein interactions. Sci Signal 2012, 5:rs6.

\section{$10.1186 /$ bcr3583}

Cite this article as: Marks: Refining the role of BRCA1 in combating oxidative stress. Breast Cancer Research 2013, 15:320 